\title{
Conducting Research at Language Centers: Practical Perspectives from the Field
}

\author{
SUSAN GASS
}

Southeast University, Nanjing, China

Michigan State University

East Lansing, Michigan, United States

\author{
ALAN JUFFS \\ University of Pittsburgh \\ Pittsburgh, Pennsylvania, United States \\ SUE STARFIELD \\ University of New South Wales \\ Sydney, Australia \\ KEN HYLAND \\ University of East Anglia \\ Norwich, United Kingdom
}

The focus in this article is on research in language centers. Four center directors provide examples of the place of research in language centers and focus on how teachers and researchers work together to facilitate large action research projects. The first section describes a survey of instructors in U.S.-based intensive English programs (IEPs). The other sections then illustrate how research is encouraged and developed through clear frameworks, incentives, and the involvement of teaching faculty in research projects of different scales. 
\& The purpose of this article is to elucidate issues that confront English language centers in four different academic contexts in three different countries. ${ }^{1}$ The focus is on how research ${ }^{2}$ is fostered and facilitated within language centers that are generally viewed by the universities in which they are housed as service units. Through this account, we hope to provide information on attitudes to research in centers and to illustrate how four directors (two from U.S.-based English language centers, one from an Australian center, and one from Hong Kong) approach the goals of promoting research in their units. As noted above, centers are often viewed as "service providers" for the university and as such they may not be expected to engage in the research activities required in other academic departments. Thus, our goal is to show how research can be promoted in language centers and to discuss frameworks and practices that promote research activity which might be models for other centers to adapt for themselves.

Research can take on many different forms, including research that involves hardcore data collection, analysis, and interpretation, and research that everyone does on a daily basis with a focus on classrooms. The former typically leads to publication, whereas the latter may or may not. Both are valid forms of investigating classroom practices and both are found in language centers around the globe.

To provide a snapshot of current involvement by teachers in U.S. intensive English programs (IEPs), we begin with a report conducted by Gass on a survey of language teachers who were asked about their attitudes toward research, the importance they place on research, and their own participation in research projects. In the second section, Juffs illustrates ways in which collaboration between teaching faculty in an English language institute and department faculty with graduate students in academic programs is facilitated. In the third part, Starfield emphasizes two different types of research; in the last section, Hyland discusses processes and types of research in his center.

\section{RESEARCH IN U.S. INTENSIVE ENGLISH PROGRAMS: FOCUS ON TEACHERS}

Gass carried out a survey of teachers in IEPs that are members of University and College Intensive English Programs (UCIEP), a U.S.-

\footnotetext{
${ }^{1}$ Space limitations do not allow detailed descriptions of the contexts described in this report. For further information about U.S.-based English language centers, the interested reader is referred to Hoekje and Stevens (2017).

${ }^{2}$ We take a broad view of the construct research,' including what might be referred to as conventional research or practitioner, action research. We recognize that different types of research may be perceived as more or less relevant and valid depending on the specific academic context. This debate goes well beyond the scope of this report.
} 
based consortium of IEPs (www.uciep.org). Rigorous standards are applied in determining UCIEP membership, including standards for teachers. IEPs are housed in different units in different universities, for example in academic departments (e.g., English, linguistics), in continuing education, or reporting directly to a provost through an international center. Thus, the context and academic support for IEP teachers and research varies greatly.

The survey elicited 49 responses, although not everyone responded to all questions. Approximately three-quarters of the respondents had been teaching in an IEP for 5 years or more with nearly $40 \%$ for 10 years or more, indicating a sample of experienced teachers.

To put their responses in perspective, their graduate school training was surveyed, offering three choices of emphasis: (1) mostly pedagogical, (2) mostly research, and (3) an equal amount of both. Nearly half reported that their training was mostly pedagogical; only $5 \%$ had mostly research training, and nearly half had an equal amount of research and pedagogy training. Knowing that research training encompasses a broad range of possibilities, Figure 1 ( $n=33$; respondents responded to as many as applicable) reflects responses to categories of research training that teachers had received. Most respondents replied in the general category, but Figure 1 suggests that qualitative and action research are both common as well.

The next section of the survey asked about instructors' specific experience with and attitude toward conducting research. When asked if it was important for faculty in intensive English programs to conduct

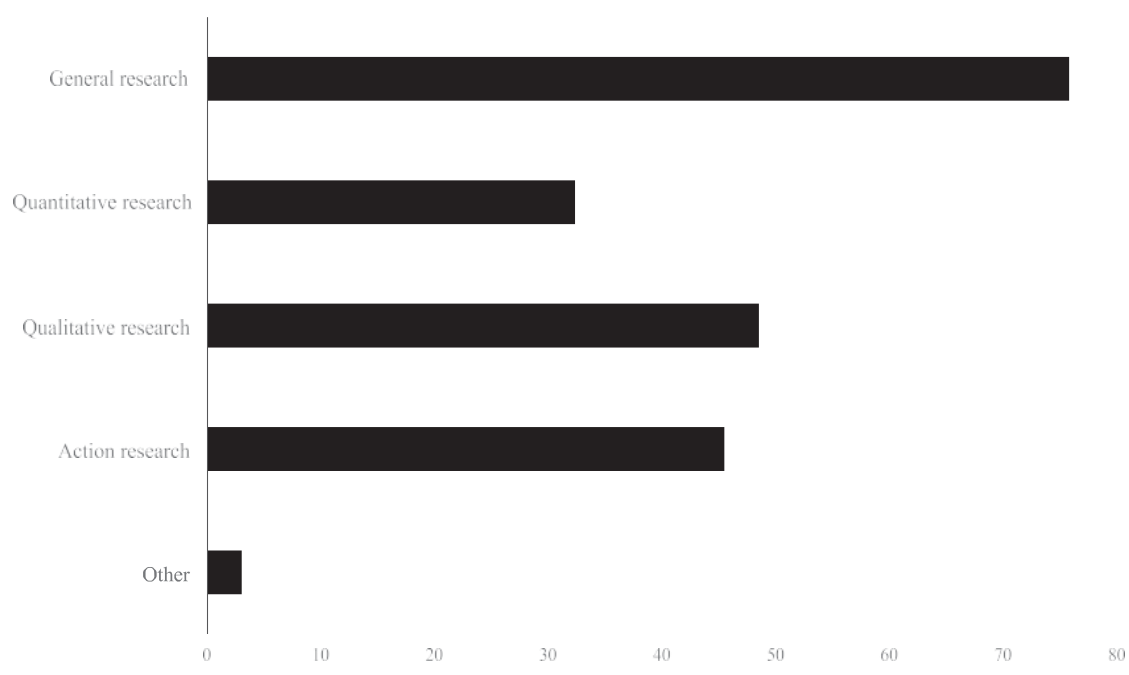

FIGURE 1. Type of training (\%). 
research, $83 \%$ said yes, $13 \%$ said maybe, and $4 \%$ said no. The same trend was found when asked if faculty should be encouraged to conduct research, although more went into the maybe category $(71 \%=$ yes; $25 \%=$ maybe; $4 \%=$ no).

Seventy-five percent had conducted research and 25\% had not. Figure 2 displays the venues of presentations and/or publications. As can be seen, in most instances research results were presented locally, although close to half presented nationally/internationally and nearly $20 \%$ have published their results.

Beyond the quantitative responses are the interesting comments received. Responses were mainly in the categories of (a) personal growth (e.g., "self-assessment," "helped improve own teaching," "feeling of accomplishment"); and (b) program benefits ("assisted others in understanding problems," "helped me to better understand the student population, and how to better serve them," "helped see a potential disconnect between student needs and curriculum," "helped my department understand student learning and possible ways of accelerating learning." Thus, teachers see research as beneficial to their own and their program's development.

Those who had conducted research also commented on their experiences, as can be seen in the following responses: "allowed me to step outside of the minutiae of day-to-day teaching," "gave me credibility," "reminded me of the need to subject my opinions to scrutiny (science is good for the mind and the soul)," "my perspective of the issue changed and it gave me more confidence in trying to convince my students of the validity of certain classroom exercises."

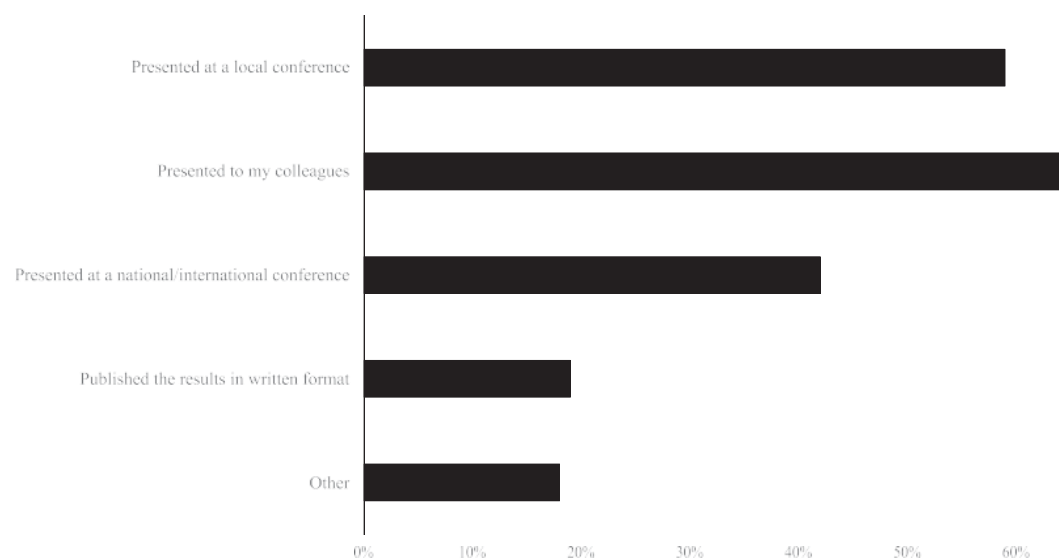

FIGURE 2. Dissemination of research results. ( $n=36$; respondents responded to as many as applicable.) 
Thus, teachers have both the training and the interest in research and apply it to their teaching. Despite the overwhelming interest in conducting research, three obstacles came up repeatedly: lack of support from the center, requirements of the job, and time. The second of these often related to the unionization of faculty. When contracts specify teaching responsibilities that add up to $100 \%$ of one's position, no time exists for research and teachers may be prevented from actively participating in research. ${ }^{3}$ With regard to time, when asked "if time were not an issue, would you be interested in conducting research?", 69\% responded that they would do research, $21 \%$ gave the response a maybe, and $10 \%$ said they would not.

These data suggest an opportunity for IEP and language center directors to take the lead in creating environments where pedagogy and research live side by side. Because so many faculty members have a strong knowledge base and an interest in conducting research, directors of English language programs are recommended to (1) allocate resources for research projects; (2) reassign duties to allow time for research; (3) provide a small amount of funding for projects (perhaps competitively); (4) conduct small workshops with those interested; (5) encourage faculty to audit research methods classes to learn more about research and as a way of getting involved with research projects; (6) set up meetings with university graduate students and research faculty to facilitate research partnerships; and (7) most importantly, discuss these issues with faculty to determine how best to facilitate the process for creating and sustaining a culture of inquiry and discussion. We turn next to three specific reports from center directors.

\section{FOUNDATIONS IN APPLIED LINGUISTICS AND COLLABORATION PROMOTE RESEARCH AT THE UNIVERSITY OF PITTSBURGH'S ENGLISH LANGUAGE INSTITUTE}

In this section, the director of an IEP and English language institute (ELI) that is embedded in a department of linguistics at the University of Pittsburgh illustrates a context where teaching and research have been able to flourish. The ELI runs an IEP with 100 to 150 full-time students, but also serves over 1,000 matriculated students in various schools of the university (e.g., Arts and Sciences, Engineering, Information Science, Law and Business). The Department of Linguistics

\footnotetext{
${ }^{3}$ By actively participating in research, we intend those activities that might lead to collecting and analyzing some data set and then presenting the results of the analysis either locally, nationally, or internationally.
} 
and the English Language Institute were established and woven together by some of the early pioneers in TESOL, Professors Edward M. Anthony and Christina Bratt Paulston. The current director is a professor of second language acquisition (SLA), has experience in teaching in different contexts, and has worked with ELI faculty to write materials based on in-house research. The associate directors are also active in research and publishing. Thus, the leadership of the unit and its close association with an academic department provides a context for research to thrive.

To facilitate research, a clear framework for carrying out formal studies has been established in the faculty handbook that creates a clear, welcoming gateway for researchers. The process is as follows: the research coordinator brings a proposal to a faculty meeting either from a professor, a graduate student, or another person who wants to carry out research from another school. The IEP faculty then approve the study so that they are "involved." (All projects require approval by the Institutional Review Board). If the proposal is a classroom-based intervention, the researcher must meet with the specific curriculum supervisor and ensure that the intervention meshes with the student learning outcomes for that curriculum. (Usually, no "control" group is possible because students talk to one another, and if an intervention is thought to be beneficial all students should be exposed. It is, however, possible to test students the semester before an intervention to obtain comparison group data.) Outside researchers from psychology, linguistics, and education are actively encouraged and welcomed through contacts of the director and other faculty members.

As a result of this collaboration and openness to research, fruitful larger projects have developed across the campus of the University of Pittsburgh and further with Carnegie Mellon University on a National Science Foundation project resulting in over 23 articles and MA and $\mathrm{PhD}$ theses since 2005. Several studies were carried out on fluency development, on an activity theory approach to cultural influences in using intelligent tutors ${ }^{4}$ for reading, and using statistical models to evaluate placement decisions.

Articles based on a large corpus of learner data have resulted in research on syntactic development, first language (L1) influences on spelling, and measures of lexical diversity and sophistication in students' writing. These research projects are reported back to the teachers through colloquia and informal talks, making the results available to the teachers who then benefit from their involvement. A list of

\footnotetext{
${ }^{4}$ An intelligent tutor is a computer program that guides learners through stages in acquisition of a skill. The tutor may provide hints and give feedback on accuracy.
} 
research projects and some papers are accessible on GitHub (https:// github.com/ELI-Data-Mining-Group/Pitt-ELI-Cor).

Professional bodies such as UCIEP and the Commission on English Language Program Accreditation (CEA) encourage professional development of teachers in language centers. In this context, we note that significant research by teachers may go unnoticed, but is vital to the success of the institute. In fact,

Teachers are conducting a form of micro-research when they make data-driven decisions about activity selection, material development, remediation, etc. These teachers distinguish themselves from others because they use student language data to inform decisions, rather than just following a provided sequence or choosing something because students will like it.

(Dawn E. McCormick, PhD, personal communication, March 14, 2017).

In this center, such activity is incentivized by providing funding to any teacher who has a paper proposal accepted at a conference. This latter point has resulted in three to five faculty members attending and presenting at international TESOL conferences in each of the past four years.

Thus, the ELI encourages both large government-funded research and smaller "action research" work by teachers. This successful culture of research depends on collaboration among administrators and with teachers across campus, connections to an active graduate program, and an enduring interest by all in the successful learning outcomes of the students. At this institute, this success derives in part from many of the recommendations by the first author already being in place.

\section{FACILITATING RESEARCH WITHIN AN AUSTRALIAN LEARNING CENTRE}

The Learning Centre (LC) at the University of New South Wales (UNSW) Sydney is not an English language center as understood in the North American context. The LC offers academic skills support to all enrolled students; given the diversity of the student body, many of those students are from non-English-speaking backgrounds

In 2016, 56,085 students were enrolled at UNSW, 30\% of whom were international students. The LC has a permanent staff of 9.4 full time equivalent (FTE) which includes 5.6 FTE learning advisers and a .8 learning resource developer. All staff apart from the director are considered professional (i.e., not academic) appointments. Learning advisers provide academic skills support to students and also work with 
academic colleagues to support students within their courses. Unlike some contracts mentioned by Gass in discussing the results of the survey conducted, no institutional requirements limit learning advisers from carrying out research. However, given the small number of learning advisers, responding to the high demand for support while fostering research and encouraging research-informed teaching constitutes a challenge. Nevertheless, this challenge can be met by drawing on two specific approaches to research, namely design-based research (DBR) and translational research.

\section{Design-Based Research}

DBR is a practitioner-oriented approach to research that supports the exploration of educational problems through an iterative "tweaking" of learning environments using feedback and reflection from learners and teachers over time to promote improvement (The DesignBased Research Collective, 2003; Kennedy-Clark, 2013). (Thanks are due here to Donald Freeman for introducing me to design-based research.) For example, the development over time of a set of online resources on report writing for engineering students from a range of departments used a DBR approach to support large numbers of students in the first-year engineering program and beyond. Initially, generic, paper-based handouts on report writing were converted to online resources. Over time these were developed and modified in response to student and faculty feedback and our in-class experience of working with engineering students. With the assistance of some grant funding, the substantial iWrite Program (http://iwrite.unsw.edu.au/iwrite.html) was built that now contains report-writing resources for both students and faculty. These interactive learning resources have been developed through careful linguistic and discourse analysis of successful reports within specific disciplinary areas as research; our experience showed that the report genre varied considerably across engineering subfields such as mining, civil, and chemical engineering. Audio recordings of interviews with faculty provide additional information for students. The facultyoriented resources are intended to assist engineering faculty to integrate the teaching of report writing into the curriculum. The Centre still does face-to-face teaching of report writing skills in large lecture classes for many engineering students. However, iWrite enables us to reach more students online to provide a level of genre and lan- guagebased support that is typically not possible in a large, one-off lecture while also providing a set of resources to embed in learning management systems across the university. 


\section{Translational Research}

The second research approach draws on a strategy frequently adopted in medical research. Translational research aims to "translate" research into practice, for example, taking basic science findings from the laboratory to applications at the patient's bedside (Woolf, 2008). Translational research makes engagement with practitioners and the wider community its priority as it seeks to "translate" research in ways that enable that research to be applied. In so doing, it seeks to "close the circle" by allowing practitioners to provide feedback to researchers based on their experience. DBR and translational research are thus not unrelated in their overall intentions. My colleague Jamie Roberts (Roberts, 2017) has recently drawn on his many years' experience of teaching essay writing, and of thinking deeply about how best to do so, in order to author a handbook that explains to students what markers of successful essays look for.

Both the translational approach to research and DBR clearly have a strong practitioner orientation, but simultaneously build and draw on continuous "tweaking" and reflection. In a context in which research is not mandated, both seem ideal vehicles for fostering a researchinformed culture.

\section{FOSTERING AND FACILITATING RESEARCH AT THE CENTRE FOR APPLIED ENGLISH STUDIES AT THE UNIVERSITY OF HONG KONG}

Research is central to both the professional development of teachers and the effective instruction of learners, and is therefore indispensable to English language centers. The problematization of pedagogy should be a matter of regular communal discourse among staff in corridors and meeting rooms, and research can inspire and inform these discussions and the courses that result from them.

The English Language Centre at the University of Hong Kong is a large and diverse center with some 70 staff and a remit to provide compulsory credit-bearing courses to 6,000 undergraduates and 1,500 research postgraduate students each year across the 10 faculties of the (English-medium) institution. In addition to these courses, the center teaches numerous self-funded programs to faculties and administrative departments around the campus, offers an MATESOL and a Master of Arts in Applied Linguistics, while individual faculty supervise 10-12 $\mathrm{PhD}$ students. Apart from five staff members, teachers are not officially required to engage in research, but the revenue from self-funded 
programs supplements our budget and allows reduced teaching loads to support further study, conference attendance, small research projects, and guest speakers for seminars.

At the heart of our research is the exploration of disciplinary practices, particularly the written genres, required of the students we teach. The reform of the Hong Kong education system in 2012, which added a year and English requirements to the 3-year university program, was the catalyst for a thorough revision of our English language curriculum. Our research thus recognizes that because the conventions of academic communication differ considerably across disciplines, identifying the particular language features, discourse practices, and communicative skills of target groups is vital to teaching English in universities. As a result of discussions with faculty members, examination of course texts and student writing, and focus groups with learners, we have discovered some weird and wonderful genres and have been able to develop and refine 30 different English-in-the-discipline courses.

Another large area of research in the center has involved identifying aspects of English which will most effectively help some 3,000 freshmen successfully bridge the gap between the English learnt at secondary school and the English required in their disciplinary studies in their second year. Our research shows that faculty in the different disciplines have certain expectations about how writing should be structured as a coherent argument, that students should take a stance which is appropriately hedged and evidenced, and have some control over features like nominalisation, impersonality, lexical density, and so on.

Many of these research projects have been initiated by program coordinators tasked with developing courses for students in different faculties, often with the support of class buy-out time funded by university Teaching Development Grants. For this to work, however, we have found it important to create an environment where research is seen to be useful and valued. Here our PhD students and assistant professors play an important role as do regular seminars by visiting speakers and, recently, hosting two major conferences. It is equally imperative, however, to engage teachers, especially junior staff who often struggle to keep up and make sense of their roles in research. We therefore encourage them to join special interest groups, contribute to regular seminars where colleagues share the results of action research or classroom projects, and participate in mandatory formative peer class observations. In these ways teachers feel they have a stake in the research going on and are, hopefully, inspired to do some themselves.

There are, of course difficulties. Contemporary ethics procedures can be dauntingly inflexible and faculty are often unwilling collaborators in our research, sometimes viewing our work with the suspicion 
we are encroaching on their disciplinary turf. We have therefore been careful to recruit their expertise while avoiding "partnerships" which leave us vulnerable to a subordinate role. Finally, research is often undervalued by institutions themselves. Around the world modern neoliberal universities are keen to raise money from fee-paying international students and cut costs by employing teachers on short contracts with heavy teaching loads. But we cannot afford NOT to do research.

\section{CONCLUDING REMARKS}

The four sections in this brief report emphasize different aspects of research language centers, which are typically viewed as service centers to their universities without a mandate of research activity. Although research is rarely "built in" to center organizational structure, all four directors have incorporated research into the ongoing life of their centers and all serve as models in that they have active research agendas of their own. We hold that research, in whatever form it takes, must constitute an essential part of such centers if personnel and curricular stagnation is to be avoided. To do this, it is important to bring teachers into the process. The fourth author speaks of teachers feeling that research projects might be viewed as encroaching on subject specialists' territory. Recognizing outside expertise, as he notes, is an important part of the path forward. And, as the first author found, most teachers do want to participate actively in research. How each center approaches research may be dependent on the director's research orientation and background, but all approaches involve collaboration. While the respect and impact our centers have within university contexts is partially dependent on the contributions the centers make locally, their standing also derives from the broader academic communities in which each teacher or researcher operates. Finally, all centers must understand and promote research within their specific context and research that approaches issues from multiple perspectives. In this way, the likelihood of incorporating many voices is increased.

\section{THE AUTHORS}

Susan Gass is a professor in the School of Foreign Languages of Southeast University in Nanjing China and University Distinguished Professor at Michigan State University where from 1990 to 2018 she was the director of the English Language Center. Her research interest is on second language learning and teaching. 
Alan Juffs has taught English as a second language (ESL) in France, China, Japan, Canada, and the United States. He is currently professor of linguistics and director of the English Language Institute at the University of Pittsburgh. His most recent research focus is using learner corpora to track language development in intensive English programs.

Sue Starfield is a professor in the School of Education at UNSW Sydney (UNSW). From 2000 to mid-2018 she was the director of the UNSW Learning Centre. Her research focuses on academic literacies, doctoral writing, writing for scholarly publication, equity and diversity in higher education, and ethnographic methodologies.

Ken Hyland is now a professor at the University of East Anglia, following several years at the University of Hong Kong. He has published over 240 articles and 27 books on academic discourse with over 38,000 Google Scholar citations. A collection of his work was recently published by Bloomsbury.

\section{REFERENCES}

Hoekje, B. J., \& Stevens, S. G. (2017). Creating a culturally inclusive campus: A guide to supporting international students. New York, NY: Routledge.

Kennedy-Clark, S. (2013). Research by design: Design-based research and the higher degree research student. Journal of Learning Design, 5, 26-32. https:// doi.org/10.5204/jld.v6i2.128

Roberts, J. Q. (2017). Essentials of essay writing: What markers look for. London, England: Palgrave.

The Design-Based Research Collective. (2003). Design-based research: An emerging paradigm for educational inquiry. Educational Researcher, 32(5-8), 35-37.

Woolf, S. (2008). The meaning of translational research and why it matters. Journal of the American Medical Association, 299, 211-213. https://doi.org/10.1001/ jama.2007.26 